\title{
An Immersive Approach based on Two Levels of Interaction for Exploring Multiple Coordinated 3D Views
}

\author{
Carlos Quijano-Chavez \\ carlos.chavez@inf.ufrgs.br \\ Institute of Informatics - UFRGS \\ Porto Alegre, RS, Brazil
}

\author{
Luciana Nedel \\ nedel@inf.ufrgs.br \\ Institute of Informatics - UFRGS \\ Porto Alegre, RS, Brazil
}

\author{
Carla M.D.S. Freitas \\ carla@inf.ufrgs.br \\ Institute of Informatics - UFRGS \\ Porto Alegre, RS, Brazil
}

\begin{abstract}
The interaction with multiple 3D visualizations in 2D conventional displays lacks usability and does not guarantee the usefulness the extra dimension provides. Immersive visualization techniques can potentially fulfill these gaps by providing 3D visualizations and novel 3D interactions simultaneously. In this paper, we propose a new approach for interacting with composite and multiple coordinated visualizations in immersive virtual environments. We use a 3D-WIMP-like concept, i.e., virtual cubes (Spaces), for encapsulating views, which the user can freely control in the virtual environment. We compared our approach with a desktop version to evaluate its performance when dealing with composed tasks.
\end{abstract}

\section{KEYWORDS}

Multiple coordinated views, Virtual reality, Immersive analytics.

\section{INTRODUCTION AND MOTIVATION}

Multiple Coordinated Views (MCV) are among the most commonly used ways of composing visualization techniques to show different perspectives of the same or potentially correlated data to facilitate insight into a complex dataset [3]. Such an approach is especially suited for visual analytics applications. Depending on the data, using multiple 2D views in conventional 2D displays demands large displays, while for 3D visualizations, such setup may not guarantee a useful tool. Earlier studies showed that the interaction with multiple 3D visualizations in 2D displays does not meet usability criteria [9]. This lack of usability could be overcome if the exploration happens in immersive environments, where the user has an extra degree of freedom for interacting with 3D visualizations [2].

Although human spatial awareness and organizational capabilities can help the analytical process performed interactively in VR, developing techniques for such environments is a challenge because they require more complex control of interaction techniques [4]. Furthermore, there is a need for interaction methods capable of achieving the functionalities of the predominant WIMP (windows, icons, menus, pointer) used for visual analysis tasks [5].

In this paper, which summarizes partial results of the M.Sc. dissertation of the first author, we present an approach for interacting with multiple coordinated views that display $3 \mathrm{D}$ visualizations. Our technique uses a virtual cube as a 3D-WIMP version (Space), inspired by Mahmood et al.'s work [7], for encapsulating each view, and two modes of interaction with the views: the macro mode for

SVR 2021, October 18-21, 2021, Gramado, RS, Brazil interacting with the Spaces, and the micro mode for interacting with the data displayed in the Space.

\section{RELATED WORK}

Several immersive analytics studies have used diverse strategies to provide multiple views regarding different "composite visualization views" (CVVs) design patterns (juxtaposition, superimposition, overloading, nesting) [3], coordination techniques (brushing, navigational slaving) [10], and settings (large displays, AR and VR environments).

We analyzed those studies and presented the survey elsewhere [8]. In summary, the studies commonly used the juxtaposition pattern followed by superimposition. We also noticed that the absence of methods and practical guidelines to use composite views in immersive analytics applications induced the development of different strategies, which showed disadvantages, especially in VR environments [1]. Our work aims at overcoming those limitations by allowing users to compose visualizations and move them for improving the scene layout, facilitating data exploration.

\section{THE SPACES APPROACH AND ITS EVALUATION}

Following the design space of composite visualization [3], where multiple "visual structures" are combined in the same "view", we designed our approach based on similar concepts. The "visual structure" is mapped to a virtual cube where it is rendered. The virtual cube is called Space. A Space is a container for one visualization only and can be manipulated similarly to an object. The objective of a Space is to facilitate the interaction across multiple visualizations (Fig. 1). It can be cloned, and its title is customized with the version number to distinguish it from the original Space. To interact with a Space, the interacting agent must be in macro mode, while to interact with the data displayed inside a Space, it must be in micro mode. The Spaces can be grabbed and overlaid to facilitate comparison of the data represented inside each one (Fig. 1-left). The two virtual hands are independent from each other: the user can grab a Space with one hand and explore its information with the other one (center). Our approach allows the exploration of Multiple Coordinated Spaces (right). Multiple coordinated Spaces are based on the coordinate interactions. Each time a Space is rotated, the linked spaces will rotate too (navigation slaving). When the data is highlighted or not, the linked data will undergo the same change, thus providing the linking-and-brushing functionality.

To evaluate the Spaces approach we performed a user study based on exploring a music dataset [6], which contains the following 


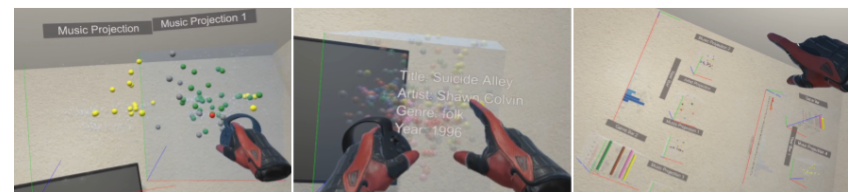

Figure 1: The Spaces approach: the macro (left) and micro (right) modes of interaction with the Spaces and the data, respectively. The controller is shown only with the macro mode. The central image shows both modes.

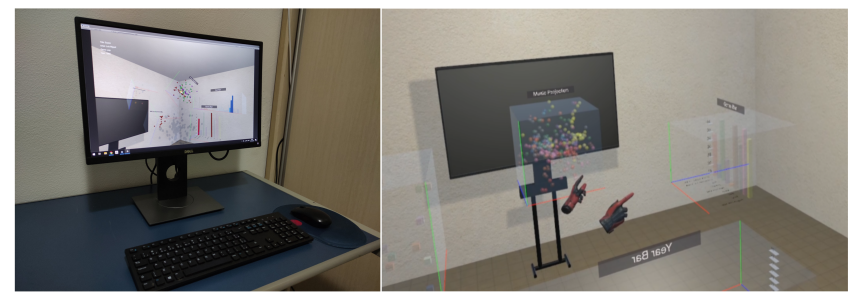

Figure 2: In the Desktop version, users interacted with the visualizations using keyboard + mouse (left), while in the VR environment they used controllers as virtual hands (right).

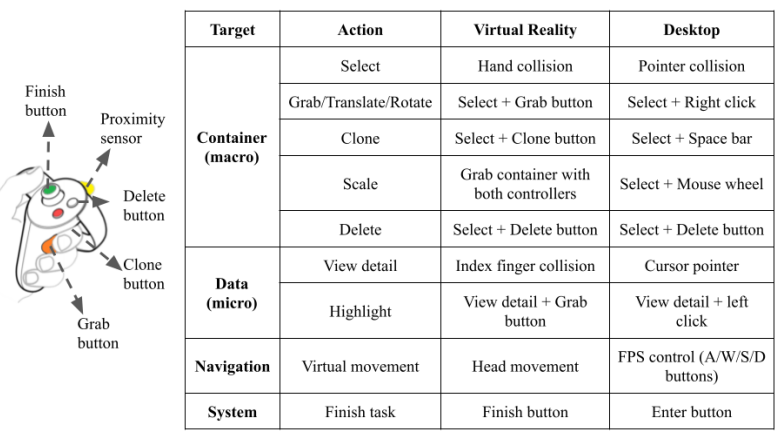

Figure 3: Distribution of actions for each interactive command used in the Virtual Reality and Desktop versions.

data for each music album: year, artist, genre, and also feature data from sound signals. A total of 338 tracks were chosen. The visualizations implemented are 3D scatterplots of music tracks, artists, and genres, obtained from a multidimensional projection technique, and bar charts showing the number of tracks per year, artist, and genre. Each visualization is displayed in a Space.

Following a within-subjects design, we compared the behavior of 19 participants (16 males, 3 females, all students) while handling 3D visualizations in Desktop and Virtual Reality.

We developed a VR-based and a similar 3D desktop version (Fig.2) with the same interactions to standardize the experiment variables (Figure 3). We used the Unity game engine, C\#, and the SteamVR plug-in to build a tool compatible with the HTC Vive and Oculus Rift head-mounted displays. The First Person navigation technique was implemented for the desktop because it is more immersive than a third-person point of view approach. We defined seven hypotheses to guide our user study and three tasks. Besides measuring time and accuracy, based on the tasks, participants answered SMEQ, UMUX-lite and Emocards questionnaires.

\section{RESULTS AND CONCLUSIONS}

The detailed analysis of our results are reported in a recent publication [8]. The results show that the Desktop version is not significantly better than the VR version in terms of time and accuracy despite using the standard FPS approach with keyboard and mouse. Multiple 3D views are not typically used in desktop versions, and this could be the reason for the non-significant results. Subjective results show that our VR approach is significantly better than the Desktop version, suggesting that the exploration of multiple 3D visualizations with common interaction devices lacks usability.

In this work, we have presented an immersive analytics approach to interact with multiple coordinated three-dimensional views in Virtual Reality. We have demonstrated the usability of our approach in a user study with non-expert participants comparing with a similar 3D desktop version. It suggests that our approach can be used in real-life scenarios. From the lessons learned with this experiment, in an on-going work we are considering to offer to the users the possibility of interacting with near objects using the virtual hand and also with far objects, through ray casting.

\section{ACKNOWLEDGMENTS}

We are deeply grateful to the subjects of our study, conducted before the COVID-19 pandemics. This work was financed by CNPq and CAPES - Finance Code 001.

\section{REFERENCES}

[1] Denis Gračanin. 2018. Immersion Versus Embodiment: Embodied Cognition for Immersive Analytics in Mixed Reality Environments. In Augmented Cognition: Intelligent Technologies, Dylan D. Schmorrow and Cali M. Fidopiastis (Eds.). Springer International Publishing, Springer, Cham, 355-368.

[2] Nicolas Greffard, Fabien Picarougne, and Pascale Kuntz. 2014. Beyond the classical monoscopic 3D in graph analytics: An experimental study of the impact of stereoscopy. In 2014 IEEE VIS International Workshop on 3DVis (3DVis). 19-24.

[3] Waqas Javed and Niklas Elmqvist. 2012. Exploring the design space of composite visualization. In 2012 IEEE Pacific Visualization Symposium. 1-8.

[4] Søren Knudsen and Sheelagh Carpendale. 2017. Multiple Views in Immersive Analytics. In IEEE VIS 2017 Workshop on Immersive Analytics. https://tinyurl. com/immersiveviews

[5] Bongshin Lee, Petra Isenberg, Nathalie Henry Riche, and Sheelagh Carpendale. 2012. Beyond Mouse and Keyboard: Expanding Design Considerations for Information Visualization Interactions. IEEE Transactions on Visualization and Computer Graphics 18, 12 (Dec 2012), 2689-2698.

[6] Dawen Liang, Haijie Gu, and Brendan O'Connor. 2011. Music genre classification with the million song dataset. Machine Learning Department, CMU (2011). https: //www.ee.columbia.edu/ \{\}dliang/files/FINAL.pdf

[7] Tahir Mahmood, Erik Butler, Nicholas Davis, Jian Huang, and Aidong Lu. 2018. Building Multiple Coordinated Spaces for Effective Immersive Analytics through Distributed Cognition. In 2018 International Symposium on Big Data Visual and Immersive Analytics (BDVA). 1-11.

[8] Carlos Quijano-Chavez, Luciana Nedel, and Carla M. D. S. Freitas. 2021. An Immersive Approach Based on Two Levels of Interaction for Exploring Multiple Coordinated 3D Views. In Human-Computer Interaction - INTERACT 2021, Carmelo Ardito, Rosa Lanzilotti, Alessio Malizia, Helen Petrie, Antonio Piccinno, Giuseppe Desolda, and Kori Inkpen (Eds.). Springer International Publishing, Cham, 493-513.

[9] C. Russo Dos Santos and P. Gros. 2002. Multiple views in 3D metaphoric information visualization. In Proceedings Sixth International Conference on Information Visualisation. 468-473.

[10] Maximilian Scherr. 2008. Multiple and coordinated views in information visualization. Trends in Information Visualization 38 (2008), 33. https://www.mmi.ifi. lmu.de/lehre/ws0809/hs/docs/scherr.pdf 